Review Article

\title{
Comparison of safety and toxicity of liposomal versus conventional Doxorubicin: an updated review
}

\author{
Rakshitha B. V.*, Nalini G. K., Sahana G. N., Deepak P., Jayashree V. Nagaral, Mohith N., \\ Divyashree C. R.
}

Department of Pharmacology, Hassan Institute of Medical Sciences, Hassan, Karnataka, India

Received: 07 March 2019

Revised: 26 March 2019

Accepted: 03 May 2019

\section{*Correspondence to: \\ Dr. Rakshitha B. V., \\ Email: dr.rakshitha7@ gmail.com}

Copyright: () the author(s), publisher and licensee Medip Academy. This is an openaccess article distributed under the terms of the Creative Commons Attribution NonCommercial License, which permits unrestricted noncommercial use, distribution, and reproduction in any medium, provided the original work is properly cited.

\begin{abstract}
Cancer persists to be a major cause of hospitalization and death every year. With the passage of time, new formulations of anticancer drugs are being introduced to the market and are drawing the concern of healthcare professionals in terms of the superiority, toxicology, and cost-effectiveness of the new formulations in comparison to the conventional formulation of the same drugs. Doxorubicin, a highly potent chemotherapeutic agent, it comes with three formulations (pegylated liposomal, nonpegylated liposomal and non-liposomal conventional formulations). English-language literature of the three formulations of Doxorubicin has been reviewed to inform the healthcare professionals regarding the differences between these formulations. Liposomal Doxorubicin promotes better toxicology profile than non-liposomal conventional Doxorubicin with an increased cost. Due to very limited studies, the cost-effectiveness of liposomal Doxorubicin is not well defined. Apart from that, this review highlights the inter patient variability in regard to the clearance and volume of distribution following the administration of liposomal Doxorubicin. In conclusion, further studies regarding the superiority of liposomal formulation of Doxorubicin, efficacy and dose standardization of liposomal Doxorubicin should be sought in the near future in a more better way.
\end{abstract}

Keywords: Cancer, Cardiotoxicity, Doxorubicin, Liposome, Pegylated liposomal formulations

\section{INTRODUCTION}

Cancer, mainly associated with the rapid and uncontrolled proliferation of cells, is the major cause of death worldwide. Cancer is the second major cause of death globally and is responsible for an estimated 9.6 million deaths in 2018. Globally, about 1 in 6 deaths is due to cancer. Approximately $180 \%$ of deaths from cancer occur in low and middle income countries. Incidence of different Cancers in the world is as shown in (Figure 1). The treatment of cancer comprises of three distinct approaches, which includes surgical excision, irradiation, and drug therapy. In case of drug therapy, side effects are almost inevitable and are also a common cause of therapeutic limitation. ${ }^{2}$

Doxorubicin is a very potent cytotoxic anticancer that directly inhibits topoisomerase II and nucleic acid synthesis, resulting in the termination of proliferation of cancer cells. However, the main limitation of the usage of Doxorubicin in anticancer treatment is its severe side effects such as cardiotoxicity like dysrhythmia and heart failure. Fortunately, this limitation could be resolved through the clinical application of liposomes. ${ }^{3}$ 

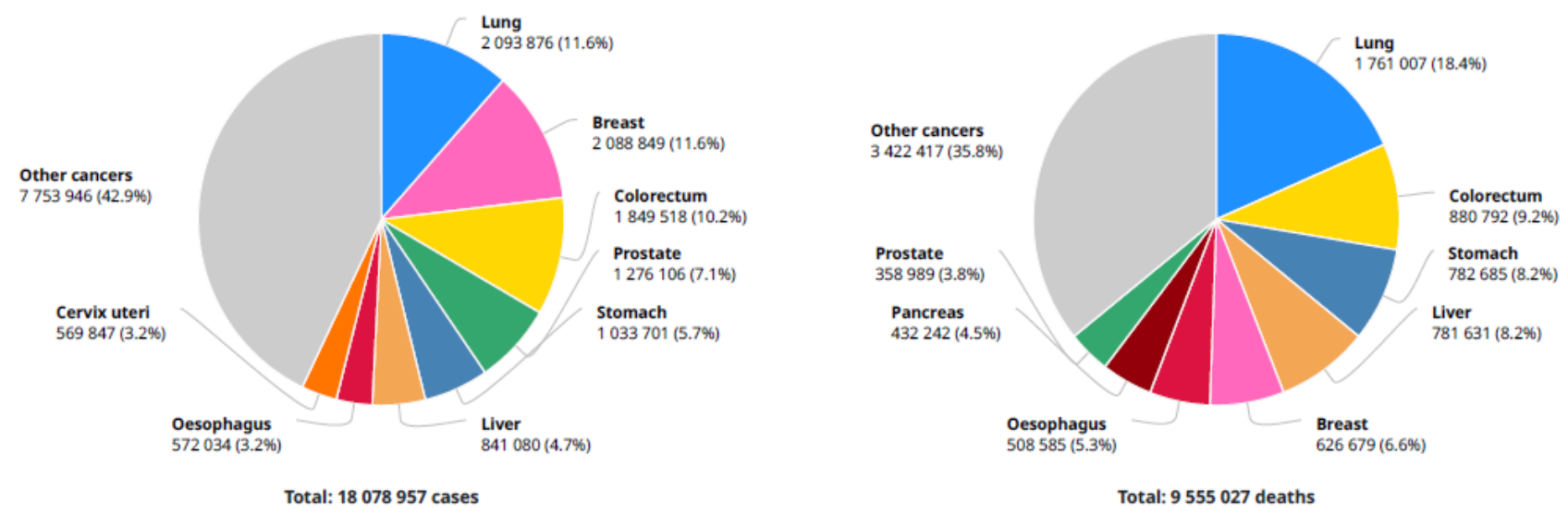

Cancer incidence and mortality statistics worldwide and by region

Figure 1: Incidence of different types of cancer. ${ }^{1}$

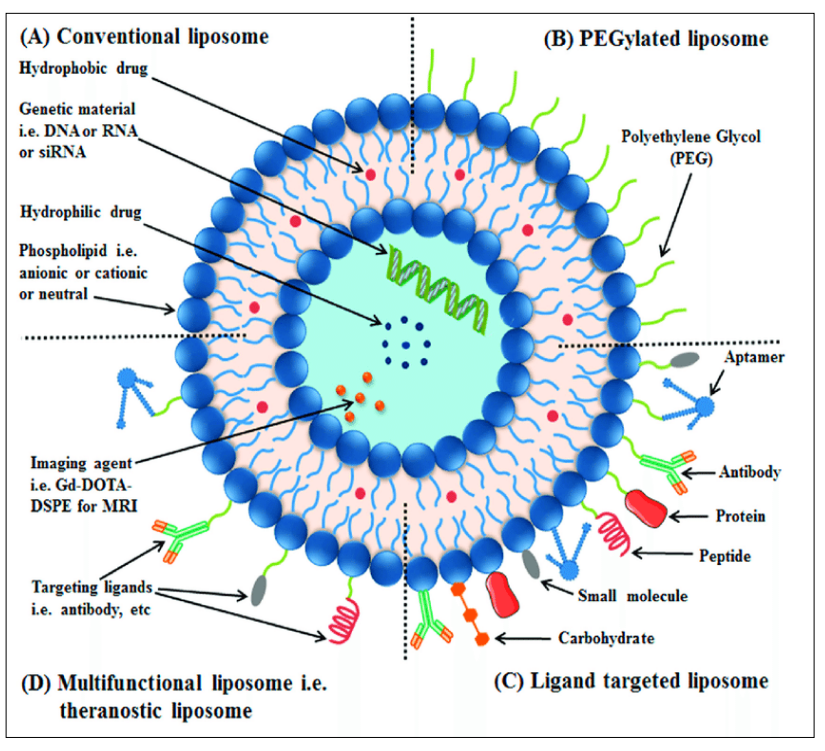

Figure 2: Liposomes: (A): Conventional liposomes are made of phospholipids, (B): PEGylated/stealth

liposomes contain a layer of polyethylene glycol (PEG) at the surface of liposomes, $(\mathrm{C})$ :Targeted liposomes contain a specific targeting ligand to target a cancer site, (D): and multifunctional such as theranostic liposomes, which can be used for diagnosis and treatment of solid tumors. ${ }^{6}$

Liposomes are bilayered phospholipid vesicles consisting of aqueous core that can encapsulate drugs. Liposomes can be easily loaded with polar and nonpolar drugs, because of their lipid bilayer and aqueous core. This makes liposomes an ideal choice for chemotherapeutics, such as Doxorubicin, and nucleic acids. Liposomes are spherical vesicles that range in size from 20 to $200 \mathrm{~nm}$ in diameter and may have one or more concentric lipid bilayers. ${ }^{4}$ In fact, liposomes can retain the drugs until being disrupted, indicating that they can promote sustained release formulation of drugs. Besides, they are also concentrated in malignant tumors, thereby enhancing the selectivity of the anticancer drugs with reduced toxicity. ${ }^{5}$ Types of liposomes are as depicted in figure.

There are several liposomal formulations of anticancer drugs authorized by United State Food and Drug Administration including Doxorubicin. A long-acting form of Doxorubicin encapsulated in liposomes has been marketed since the mid-1990s for the treatment of various malignancies. This liposomal formulation contains polyethylene glycol (PEG) coated-liposomal Doxorubicin, which is capable of targeting Doxorubicin to tumor sites. At present scenario, liposomal Doxorubicin is a therapeutic option in the treatment of AIDS-related Kaposi's sarcoma, metastatic breast cancer, advanced ovarian cancer, and relapsed/refractory multiple myelomas. ${ }^{7}$ To investigate the differences among the formulations of Doxorubicin in vivo, a literature search is conducted. It is inferred that liposomal Doxorubicin encompasses increased efficacy and better toxicology profile compared to non-liposomal conventional Doxorubicin.

\section{Pharmacological action of doxorubicin}

Although the exact mode of action of Doxorubicin remains unknown, the potency of Doxorubicin is believed to be associated with topoisomerase II, which is a DNA gyrase and is responsible for the relaxation of supercoiled structure of DNA during transcription. Specifically, Doxorubicin intercalates in the DNA and stabilizes the DNA-topoisomerase II complex during the transcription process thus prevents the relaxation of the DNA double helix and promotes termination of the process. 
Nevertheless, therapeutic limitations of Doxorubicin involve severe adverse effects such as dysrhythmia, heart failure, leukocytopenia, moderate to severe nausea, and vomiting and hemorrhage. Its cardiotoxicity such as dysrhythmia and heart failure arises from the formation of cytotoxic free radicals in the heart tissue.

Therefore, this problem can be resolved by increasing the specificity of Doxorubicin through the utilization of liposomes ${ }^{8,3}$

\section{Clinical Application of liposomes in chemotherapy of Cancer}

Liposomes features an aqueous core, one or more phospholipid membranes with/without coating groups on the surfaces of the membranes. These amphiphilic characteristics allow liposomes to carry both hydrophobic and hydrophilic drugs within the lipophilic bilayer or aqueous compartment. For instance, hydrophilic drugs dissolve in the aqueous core or adsorb on the hydrophilic head of the phospholipid bilayer whereas lipophilic drugs are filled with the hydrophobic tails of the bilayer.

There are numerous liposome-based anticancer agents being marketed as a liposomal preparation.

\section{Liposomal formulation of doxorubicin}

Doxorubicin is the best known and most widely used member of the anthracycline antibiotic group of anticancer agents. It was first introduced in the 1980s, and since that time, it has become one of the most commonly used drugs for the treatment of both hematological and solid tumors.

Table 3: List of clinically approved liposomal drugs., 9,10

\begin{tabular}{|c|c|c|c|}
\hline Name & $\begin{array}{l}\text { Route of } \\
\text { administration }\end{array}$ & Dosage Form & Indication \\
\hline Liposomal amphotericin B & Parenteral & Injectable liquid & Fungal infections \\
\hline Liposomal cytarabine & Parenteral & Injectable liquid & Malignant lymphomatous meningitis \\
\hline Liposomal daunorubicin & Parenteral & Injectable liquid & HIV-related Kaposi’s sarcoma \\
\hline Liposomal doxorubicin & Parenteral & Injectable liquid & $\begin{array}{l}\text { Combination therapy with cyclophosphamide in } \\
\text { metastatic breast cancer, Multiple Myeloma, } \\
\text { Ovarian cancer }\end{array}$ \\
\hline Liposomal IRIV vaccine & Parenteral & Injectable liquid & Hepatitis A \\
\hline Liposomal IRIV vaccine & Parenteral & Injectable liquid & Influenza \\
\hline Liposomal verteporfin & Parenteral & Injectable liquid & $\begin{array}{l}\text { Age-related macular degeneration, pathologic } \\
\text { myopia, ocular histoplasmosis }\end{array}$ \\
\hline Micellular estradiol & Parenteral & Injectable liquid & Menopausal therapy \\
\hline
\end{tabular}

The primary aim of Doxorubicin encapsulation in liposomes is to decrease nonspecific organ toxicity. Liposomes are able to drive the Doxorubicin away from sites with tight capillary junctions such as the heart muscle. Instead, they distribute in areas where fenestrations or gaps exist in the vasculature (liver, spleen, and bone marrow, areas of inflammation and neoplasms). Phagocytic cells that comprise the mononuclear phagocyte system (MPS) can recognize these particulate carrier systems as "foreign". Although distribution of these phagocytic cells is dependent on the physical (size) and chemical (charge) attributes of the liposomes used, it should be noted that when liposomes contain Doxorubicin, the cells of the MPS are adversely affected. ${ }^{11}$

More specifically, following uptake into a phagocytic cell, release of Doxorubicin causes these cells to die, thus reducing the capacity of the MPS to accumulate the injected liposomes. This is reflected, in turn, by significantly increased liposome circulation lifetimes.

\section{PHARMACOKINETICS}

Non liposomal conventional Doxorubicin 50 to $85 \%$ of plasma Doxorubicin is bound to protein. Apparent volumes of distribution are in the range of 20 to $30 \mathrm{~L} / \mathrm{kg}$ (1400 to $3000 \mathrm{~L}$ ). Doxorubicin does not cross the bloodbrain barrier. ${ }^{13}$ Doxorubicin is rapidly metabolised into the hydrophilic 13-hydoxyl metabolite, Doxorubicinol, and the poorly water-soluble aglycones, Doxorubicinone and 18-deoxyDoxorubicinone. Like Doxorubicin, Doxorubicinol is cytotoxic, but Doxorubicinone is not. Metabolism to Doxorubicinol occurs by cytoplasmatic NADPH-dependent aldoketoreductases, present in all cells, but particularly in red cells, and liver and kidney cells. This enzymatic reduction of doxorubicin is of paramount importance, as it finally produces the $\mathrm{OH}$ radicals, which causes extensive cell damage and cell death. Half-life of both Doxorubicin and doxorubicinol was 30 hours. ${ }^{14}$ Doxorubicin and its catabolites are primarily excreted in the bile. Over $50 \%$ is eliminated during the first transit through the liver. Cumulative faecal 
excretion over 18 days has been estimated at 25 to $45 \%$. Although patients often notice a reddish colouration of the urine during the first hours to days after doxorubicin administration, elimination will be around 0.18 to $23 \%$ only.

Large area under the curve (AUC), slow clearance rate (CL), small distribution volume (VD), and long elimination half-time $\left(\mathrm{t}^{1 / 2}\right)$ characterize the pharmacokinetics (PK) of pegylated liposomal Doxorubicin (PLD). ${ }^{15-18}$ The VD of PLD is close to the blood volume so that the PK of PLD undergoes single compartment model. The pegylated lipids in the liposomes result in a long circulation half-time, typically 3-4 days. ${ }^{15,16}$

Non liposomal conventional Doxorubicin consists of a large VD indicating that a significant amount of the drug is taken up in normal tissues. ${ }^{15,18}$ Apart from that, the AUC for conventional nonliposomal Doxorubicin is about three orders of magnitude smaller than PLD resulting in a CL rate about three orders of magnitude larger. The $t^{1 / 2}$ for conventional Doxorubicin is about 20-25 h. ${ }^{15,16}$

liposomal Doxorubicin has a shorter $\mathrm{t}^{1} / 2$ than PLD but a longer $\mathrm{t}^{\mathrm{t}} / 2$ than conventional nonliposomal Doxorubicin. ${ }^{18}$ It is due to the absence of PEG coating in the formulation, which indicates that it can be easily taken up by the reticuloendothelial system (RES) and can undergo metabolism. ${ }^{19}$

Despite the fact that there are imperative benefits associated with PLD and non-PLD than nonliposomal conventional Doxorubicin, its interpatient variability in terms of PKs are more clinically significant in comparison to conventional nonliposomal Doxorubicin. ${ }^{15,16}$

Regarding the nonliposomal conventional Doxorubicin, factors contributing to interpatient variability are hepatic impairment, patient age and polymorphism in efflux transporter and metabolizing enzymes. Doxorubicin is hepatically cleared by carbonyl reductases (CBR) and cytochrome P-450 enzymes, especially CBR1, CBR3, CYP3A4, CYP2C9, and CYP2D6, which implies that genetic polymorphism of CBR affects the CL of Doxorubicin. In relation to that, patients with hepatic impairment as well as elderly patients are less capable to metabolize Doxorubicin due to their insufficient metabolizing enzymes of Doxorubicin. Apart from that, a various subfamily of ATP-binding cassette $(\mathrm{ABC})$ is responsible for pumping out Doxorubicin, including $\mathrm{ABCB} 1, \mathrm{ABCB} 5, \mathrm{ABCB} 8, \mathrm{ABCC} 5$, and $\mathrm{ABCG} 2$. Provided that, polymorphism of the efflux transporter $\mathrm{ABC}$ can positively or negatively impact the plasma concentration of Doxorubicin. ${ }^{20}$

In comparison to conventional non liposomal Doxorubicin, PLD and liposomal Doxorubicin undertake a more complicated metabolizing pathway. Theoretically, the CL of liposomes depends upon the RES, involving monocytes, macrophages, and dendritic cells. Hence, besides the metabolism of Doxorubicin in the aqueous core, the CL of both PLD and non-PLD bears upon the immune system as well as the RES function of different individuals. ${ }^{16}$ Deterioration of immunity is common among elderly population, which is scientifically known as immunosenescence. Hence, the CL of Doxorubicin in an elderly patient is further reduced which may possibly prolong $\mathrm{t}^{1 / 2}$ and AUC of Doxorubicin. In spite of the unclear reason, gender is discovered to be an important contributing factor for the CL of liposomal Doxorubicin. Clinical significantly, female patients have a lower CL of liposomal Doxorubicin than male patient. ${ }^{15}$ Although the exact reason for this phenomenon remains unknown, it is thought to be closely associated with the hormone. As hormone plays a key role in the immunosuppressive and immunostimulatory activity, the reason behind this observation can be rationalized. ${ }^{21}$ There are many factors contributing to the immune status of individuals, indicating the dramatic interpatient variability of liposomal Doxorubicin.

Other factors contributing to interpatient variability of PLD are body fat composition and genetic viability. ${ }^{22}$ The phenomenon of significantly increased AUC of PLD due to high intraabdominal fat content had been observed. In terms of genetic viability, higher VD and CL rate has been detected in Asian in comparison to European. ${ }^{23}$

\section{Efficacy}

The efficacy of the different formulations which involve Doxorubicin was evaluated based on response rate, including complete response, partial response, and overall response. The survival rate, which includes overall survival and progression-free survival, is also deemed to be an indicator of efficacy.

The efficacy of PLD as a single agent in the treatment of metastatic breast cancer has been confirmed. However, there is a lack of scientific consensus that the liposomal formulations of Doxorubicin increase the survival rate of the treatment, in comparison to nonliposomal conventional Doxorubicin. Nevertheless, it is clinically significant that PLD decreases the risk of fatal cardiac events such as acute myocardial infarction and congestive heart failure. As a result, the utilization of PLD increases the survival rate of patients with high cardiac risks in comparison to nonliposomal conventional Doxorubicin.

Despite the efficacy of Doxorubicin in the treatment of glioma in vitro, its utilization is limited by the efflux effect of the blood-brain barrier (BBB). Fortunately, the development of liposomal Doxorubicin allows penetration of Doxorubicin into the malignant glioma cells in the brain. In spite of the fact that PLD shows high potency in the treatment of glioma, its dosing regimen in children remains unclear. Hence, further studies are required to balance the toxicology profile and efficacy of PLD in the treatment of glioma. ${ }^{24}$ 
In addition, laboratory data have showed the efficacy of PLD in the treatment of intracranial model of breast cancer in mice. In this model of breast cancer, PLD promotes higher survival rate and efficacy with reduced toxicity than nonliposomal Doxorubicin in mice. Clinical data regarding the utilization of PLD in this model of metastasis breast cancer is yet to be released.

\section{Toxicology}

In comparison to nonliposomal conventional Doxorubicin, it is certain that the liposomal formulations of Doxorubicin promote better cardiac safety. The reduced cardiac toxicity has also been observed in comparison to other anthracycline-based chemotherapy. Therefore, it is recommended that the liposomal formulations of Doxorubicin should be used in the patients with high risk of cardiac events such as arrhythmia, congestive heart failure, and myocardial infarction.

Furthermore, the reduced toxicity has also been observed in terms of myelosuppression and infection in comparison to nonliposomal conventional Doxorubicin. However, the myelotoxicity of liposomal Doxorubicin is not common. The myelotoxic effects in association with liposomal Doxorubicin include leukopenia, neutropenia, thrombocytopenia, and febrile neutropenia.

In terms of extra-myelotoxicity other than cardiotoxicity, the occurrence of Palmar-Plantar Erythrodysesthesia (commonly known as a hand-foot syndrome) is similar in both liposomal and nonliposomal formulations of Doxorubicin. Nausea and vomiting are moderate to severe in patients treated with nonliposomal Doxorubicin but are usually mild in patients treated with liposomal Doxorubicin.

As the liposomal formulations of Doxorubicin undergo viability in relation to $\mathrm{PKs}$, dose-dependent myelotoxicity cannot be effectively predicted. Factors affecting the PKs of liposomal Doxorubicin are likely to affect the toxicology profile of such formulations. In general, higher risk of toxicity is expected to be seen in elderly patient, immunosuppressive" and female individuals. ${ }^{15}$ Patients with high body fat composition, particularly intraabdominal fat, are also more susceptible to experience Doxorubicin-associated myelotoxicity if they are treated with liposomal Doxorubicin. ${ }^{22}$ Under the extremely rare scenario, acute peculiar mucous reaction following administration of PLD had been reported. No study had been conducted in this area as the occurrence of this reaction had not been observed prior to the case report. Hence, further study has to be carried out in this area.

\section{DISCUSSION}

This review highlights the clinical significance associated with the use of liposomal Doxorubicin. It is impacted by age, gender, race, immune status, and body fat composition of an individual treated with liposomal
Doxorubicin. An important clinical concern is that most cancer patients are middle-aged or elderly, indicating a need for dose adjustment in the treatment of liposomal Doxorubicin. Otherwise, the dose-dependent toxicity associated with liposomal Doxorubicin cannot be extrapolated and managed. However, the effective way of individualizing the dose of liposomal Doxorubicin has not been identified yet.

In the near future, the liposomal Doxorubicin will be prescribed in more conditions such as pediatric glioma and intracranial model of breast cancer as the utilization of the liposome brings about the penetration across BBB. As the PK model of the liposomal Doxorubicin in children remains unclear, more comprehensive precautions will be required to prevent or manage the adverse drug reaction of the liposomal Doxorubicin in this population. Concerning the intracranial model of breast cancer, further studies are needed to investigate how the liposomal Doxorubicin behaves in human setting. ${ }^{23}$ In terms of efficacy, there is limited evidence base to support the superiority of the liposomal Doxorubicin in comparison to the nonliposomal conventional Doxorubicin.

Nevertheless, special precautions are recommended before choosing a formulation of Doxorubicin for high-risk patients to protect against fatal cardiac events, as the reduced cardiotoxicity promoted by the liposomal Doxorubicin has been confirmed. Subsequently, the clinical concern that appear is the cost-effectiveness of the routine cardiac surveillance prior to the introduction of liposomal Doxorubicin. Overall, there remains a considerable controversy over the relative importance of routine cardiac surveillance in the patients accepting Doxorubicin-based therapy.

Although the updated cost-effectiveness of the liposomal Doxorubicin compared to nonliposomal Doxorubicin remains unclear, the cost-effectiveness of liposomal Doxorubicin in comparison to other chemotherapy is within the Willingness to Pay (WTP) threshold in most developed countries, so that the use of liposomal Doxorubicin is deemed to be cost-effective only in this particular countries. In healthcare settings, liposomal Doxorubicin is considered to be more tolerable than nonliposomal conventional Doxorubicin in regards of cardiotoxicity, myelotoxicity, nausea and vomiting with an estimation of 100 times the additional cost. Therefore, further pharmacoeconomic studies comparing liposomal and nonliposomal formulations of Doxorubicin will be required to confirm the cost-effectiveness of liposomal Doxorubicin.

In addition, this review reveals some limitations and weaknesses in relation to the updated evidence. The lack of blinding and allocation concealment in the randomized control trials could probably lead to a bias toward the superiority of liposomal formulation of Doxorubicin compared to nonliposomal conventional Doxorubicin. Another common weakness in most of the literature is the 
underpowered sample size. Therefore, it is identified that the sample present in the studies may not represent the whole population. Hence, larger studies are required to confirm the actuality of the results. Notwithstanding, the contamination, and co-intervention in most of the studies are well controlled, indicating that the results could be statistically and clinically significant. Further, our review did not compare the efficacy and toxicology of liposomal Doxorubicin with other marketed chemotherapy, which is thought to be closely related to the current healthcare settings.

\section{CONCLUSION}

There are several advantages of using liposomal doxorubicin. These include prolonged half-life, increased drug stability and selective accumulation in tumor, which reduce adverse effects to the heart. The promising results with liposomal doxorubicin in cancer has created a rationale to expand their use, but this should be approached with care, taking in consideration pharmacoeconomic aspects. In addition, the long-term safety issues of the liposomal formulation should be carefully evaluated before their routine application for the curative treatment of early stage disease.

Funding: No funding sources Conflict of interest: None declared

Ethical approval: Not required

\section{REFERENCES}

1. WHO 2018. Facts sheets on cancer from IARC. Available at: https://www.who.int/news-room/factsheets/detail/cancer

2. Taylor K, Aulton M. Aulton's pharmaceutics: The Design and Manufacture of Medicines. $4^{\text {th }}$ ed. United Kingdom: Churchill Livingstone; 2013:790-795.

3. Rang HP, Maureen DM. Rang \& Dale's Pharmacology. $7^{\text {th }}$ ed. United Kingdom: Churchill Livingstone; 2012:673-688.

4. Large DE, Soucy JR, Hebert J, Auguste DT. Advances in Receptor-Mediated, Tumor-Targeted Drug Delivery. Adv Therapeutics. 2019 Jan;2(1):1800091.

5. Pathak N, Pathak P. Applications liposome in cancer drug delivery and treatment: A review. Asian J Pharmaceutical Res Develop. 2019 Feb 15;7(1):62-5.

6. Riaz MK, Riaz MA, Zhang X, Lin C, Wong KH, Chen $\mathrm{X}$, et al. Surface Functionalization and Targeting Strategies of Liposomes in Solid Tumor Therapy: A Review. Int J Mol Sci. 2018 Jan 9;19(1).pii: E195.

7. Duggan ST, Keating GM. Pegylated liposomal Doxorubicin: A review of its use in metastatic breast cancer, ovarian cancer, multiple myeloma and AIDSrelated Kaposi's sarcoma. Drugs. 2011;71(18):253158.

8. Allen TM, Cullis PR. Liposomal drug delivery systems: From concept to clinical applications. Adv Drug Deliv Rev. 2013;65(1):36-48.
9. Pawar HR, Bhosale SS, Derle ND. Use of liposomes in cancer therapy: a review. Int J Pharmaceutical Sci Res. 2012 Oct 1;3(10):3585.

10. Berger JL, Smith A, Zorn KK, Sukumvanich P, Olawaiye AB, Kelley J, et al. Outcomes analysis of an alternative formulation of PEGylated liposomal doxorubicin in recurrent epithelial ovarian carcinoma during the drug shortage era. Onco Targets Ther. 2014 Aug 8;7:1409-13.

11. Abraham SA, Waterhouse DN, Mayer LD, Cullis PR, Madden TD, Bally MB. The liposomal formulation of doxorubicin. Methods Enzymol. 2005;391:71-97.

12. Bally MB, Nayar R, Masin D, Hope MJ, Cullis PR, Mayer LD. Liposomes with entrapped doxorubicin exhibit extended blood residence times. Biochim Biophys Acta. 1990 Mar 30;1023(1):133-9.

13. Speth PA, van Hoesel QG, Haanen C. Clinical pharmacokinetics of doxorubicin. Clin Pharmacokinet. 1988 Jul;15(1):15-31.

14. Bachur NR, Steele M, Meriwether WD, Hildebrand RC. Cellular pharmacodynamics of several anthracycline antibiotics. J medicinal chem. 1976 May;19(5):651-4.

15. La-Beck NM, Zamboni BA, Gabizon A, Schmeeda H, Amantea M, Gehrig PA, et al. Factors affecting the pharmacokinetics of pegylated liposomal Doxorubicin in patients. Cancer Chemother Pharmacol. 2012;69(1):43-50.

16. Boers-Sonderen MJ, van Herpen CM, van der Graaf WT, Desar IM, van der Logt MG, de Beer YM, et al. Correlation of toxicity and efficacy with pharmacokinetics (PK) of pegylated liposomal Doxorubicin (PLD) (Caelyx $\left.{ }^{\circledR}\right)$. Cancer Chemother Pharmacol. 2014;74(3):457-63.

17. Anders CK, Adamo B, Karginova O, Deal AM, Rawal S, Darr D, et al. Pharmacokinetics and efficacy of PEGylated liposomal Doxorubicin in an intracranial model of breast cancer.PLoS.one.2013;8(5):e61359.

18. Wasle I, Gamerith G, Kocher F, Mondello P, Jaeger T, Walder A, et al. Non-pegylated liposomal Doxorubicin in lymphoma: patterns of toxicity and outcome in a large observational trial. Annals of hematol. 2015 Apr 1;94(4):593-601.

19. Larbi A, Rymkiewicz P, Vasudev A, Low I, Shadan NB, Mustafah S, et al. The immune system in the elderly: A fair fight against diseases? Aging Health. 2013;9(1):35-47.

20. Monk BJ, Herzog TJ, Kaye SB, Krasner CN, Vermorken JB, Muggia FM, et al. Trabectedin plus pegylated liposomal doxorubicin in recurrent ovarian cancer. J Clin Oncol. 2010;28(19):3107-14.

21. Crivellari D, Gray KP, Dellapasqua S, Puglisi F, Ribi $\mathrm{K}$, Price KN, et al. Adjuvant pegylated liposomal doxorubicin for older women with endocrine nonresponsive breast cancer who are NOT suitable for a standard chemotherapy regimen: The CASA randomized trial. Breast. 2013;22:130-7.

22. Wong AL, Seng KY, Ong EM, Wang LZ, Oscar H, Cordero MT, et al. Body fat composition impacts the hematologic toxicities and pharmacokinetics of 
doxorubicin in Asian breast cancer patients. Breast Cancer Res Treat. 2014;144(1):143-52.

23. $\mathrm{Xu} \mathrm{L,} \mathrm{Wang} \mathrm{W,} \mathrm{Sheng} \mathrm{YC,} \mathrm{Zheng} \mathrm{QS.}$ Pharmacokinetics and its relation to toxicity of pegylated-liposomal doxorubicin in Chinese patients with breast tumours. J Clin Pharm Ther. 2010;35(5):593-601.

24. Ngan YH, Gupta M. A comparison between liposomal and nonliposomal formulations of Doxorubicin in the treatment of cancer: An updated review. Arch Pharma Pract. 2016 Jan 1;7(1):1-13.

Cite this article as: Rakshitha BV, Nalini GK, Sahana GN, Deepak P, Nagaral JV, Mohith N, et al. Comparison of safety and toxicity of liposomal versus conventional Doxorubicin: an updated review. Int $\mathbf{J}$ Basic Clin Pharmacol 2019;8:1453-9. 